\title{
Silicon-based III-V Quantum Dot Materials and Dsevices
}

\author{
Huiyun Liu \\ Department of Electronic and Electrical Engineering, University College London, London WC1E 7JE, UK \\ Authore-mail address: huiyun.liu@ucl.ac.uk
}

\begin{abstract}
III-V Quantum-dot (QD) materials and lasers directly grown on Si platform are the most prospective candidate to realize on-chip optical sources for Si photonics. The recent progress made in this field are discussed.
\end{abstract}

OCIS codes: (230.5590) Quantum-well, -wire and -dot devices; (250.5300) Photonic integrated circuits

\section{Introduction}

To meet the requirement of increased data throughput between silicon processors in modern information processing Si photonics has been under intensive investigation in recent years. As Group-IV materials have the indirect bandgap, the abundant non-radiative recombinations make Group-IV materials to be inefficient for the optical light sources. On the other hand, III-V compounds have direct bandgap, and hence have been utilized with a wide range of highperformance emitters, such as laser diodes. The integration of III-V laser diodes on Si platform will, thus be the most practical way of obtaining on-chip light sources on Si platform. In addition, in comparison with the well-established wafer bonding integration technologies, monolithic growth of III-V epitaxy materials on Si platform is more promising for low-cost and high dense integration in the long term. Self-assemble III-V quantum dots (QDs) with threedimensional carrier confinement have been intensively promoted in order to achieve high-performance III-V laser devices, in term of better defects tolerance, lower threshold current density and temperature-insensitive operation $[1,2]$. Very recently, the pioneering work of monolithic integration of III-V QD lasers on Si substrates have been demonstrated [1-8]. In this paper, we will present the growth of III-V quantum dot materials and devices on Ge, Geon- $\mathrm{Si}$, and $\mathrm{Si}$ substrates for silicon photonics [1-8].

\section{III-V QD lasers grown on Ge, and Ge-on-Si substrates}

An alternative to direct growth of GaAs on $\mathrm{Si}$ substrate is to use an intermediate epitaxial layer which creates nearGaAs lattice constant but has few defects. Because Ge is very closely lattice-matched (only $0.08 \%$ mismatch) to GaAs, Ge-coated $\mathrm{Si}(\mathrm{Ge} / \mathrm{Si})$ layers were widely employed as a "virtual substrate" for subsequent GaAs growth. Therefore, the growth of III-V QDs on Ge was first exploited. Ge and Ge-on-Si substrates (100)-orientated with $6^{\circ}$ offcut towards the [111] planes were used in our experiments. The thickness of the Ge wafer was $350 \mathrm{~mm}$. The substrate temperature was then increased to $650{ }^{\circ} \mathrm{C}$ and held at that temperature for $20 \mathrm{~min}$. The Ge substrate was then cooled to $380{ }^{\circ} \mathrm{C}$ for the growth of III-V epitaxial layers. The Ga prelayer was used for the initial nucleation of GaAs buffer layer. To ensure total Ga coverage on the Ge substrate, 1.08-monolayer Ga was first deposited. After deposition of Ga prelayers, 20 monolayers of GaAs were grown by migration-enhanced epitaxy using alternating Ga and As4 beams, followed by the addition of the III-V buffer layer at higher temperature. QD laser devices containing five InAs/InGaAs dot-inwell (DWELL) layers were then grown at optimized conditions as on GaAs substrates, with each layer consisting of 3.0 monolayers of InAs grown on $2 \mathrm{~nm}$ of $\operatorname{In} 0.15 \mathrm{Ga} 0.85 \mathrm{As}$ and capped by $6 \mathrm{~nm}$ of $\mathrm{In}_{0.15} \mathrm{Ga} 0.85 \mathrm{As}$. The InAs/GaAs quantum-dot lasers grown on Ge substrate shave been demonstrated with lasing at $1305 \mathrm{~nm}$ at room temperature and lasing operation between 20 and $60^{\circ} \mathrm{C}$ [1]. To form the Ge-on-Si virtual substrate, 2- $\mu \mathrm{m}$ Ge layer was grown using chemical vapour deposition on phosphorus-doped (100)-oriented Si substrates with a $6^{\circ}$ offcut toward [111] planes. The threading dislocation density in Ge epitaxial layer is about $5 \times 10^{6} / \mathrm{cm}^{2}$. The optimized InAs/GaAs DWELL laser structures were fabricated on Ge/Si. The cw lasing was demonstrated for the devices on Ge-on-Si substrates [7].

\section{III-V QD lasers directly grown on Si substrates}

InAs/GaAs QD laser structures were directly grown on phosphorus-doped Si substrates by a solid-source molecular beam epitaxy (MBE) system. The (001)-silicon wafer with $4^{\circ}$ miscut-angle oriented towards the [011] plane was used to suppress antiphase domains (APDs). The combined strategies of an AlAs nucleation layer, InGaAs/GaAs dislocation filter layers (DFLs), in situ thermal annealing and using QDs as active regions are applied to realize the high quality GaAs-on-Si laser structure with low defects [2]. Figure 1 (a) shows light-current-voltage (LIV) measurement of this high performance QD laser on Si with a low threshold current density of $62.5 \mathrm{~A} / \mathrm{cm}^{2}$ and a roomtemperature output power exceeding $105 \mathrm{~mW}$ under c.w. operation. The maximum lasing operation is up to $120^{\circ} \mathrm{C}$ 
under pulsed condition. Figure. 1(b) shows the ageing data for this InAs/GaAs QD laser on silicon. The device was aged in auto current control mode at $26{ }^{\circ} \mathrm{C}$ under $210 \mathrm{~mA}$ of constant applied current. A sub-linear mode model is employed to fit the aging and an extrapolated lifetime of over 100,158 $\mathrm{h}$ was determined [2].
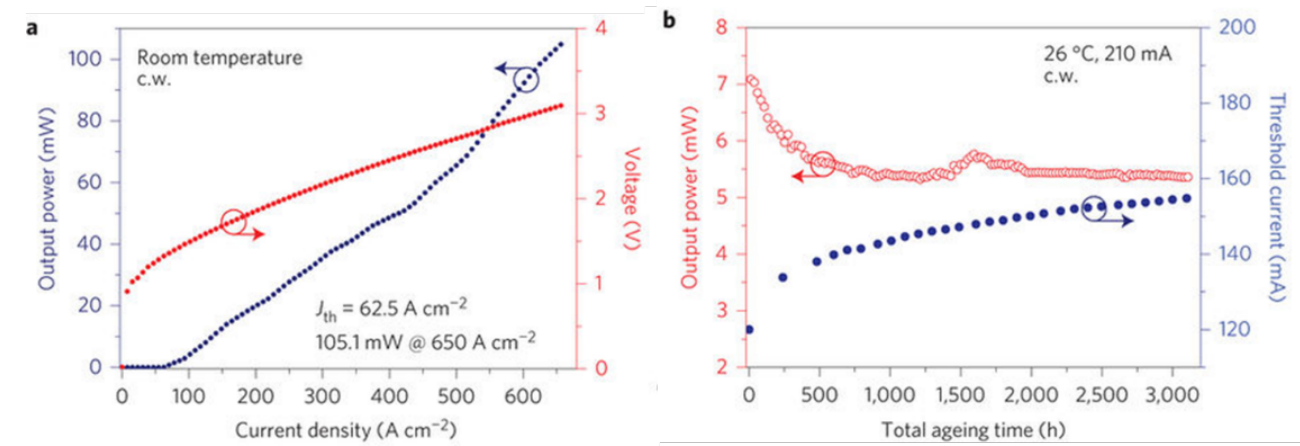

Fig. 1 (a). LIV curve of InAs/GaAs QD lasers on off-cut Si substrate under c.w. operation at room temperature; (b) Aging test of Si-based QD laser at a constant heatsink temperature of $26^{\circ} \mathrm{C}$ and $210 \mathrm{~mA}$ driving current.

\section{Conclusion}

In conclusion, we have demonstrated the practical InAs/GaAs QDs lasers on $\mathrm{Ge}$, Ge-on-Si, and $\mathrm{Si}$ substrates with high power and long lifetime at $1300 \mathrm{~nm}$. These results could ultimately form the basis for the monolithic integration of 1300-nm InAs/GaAs QD lasers on Si platform, as well as for the integration of other III-V devices on Si substrates in order to realize the long-dreamed of III-V/Si optoelectronic integrated circuit.

[1] H. Liu, T. Wang, Q. Jiang, Q. Hogg, F. Tutu, F. Pozzi, and A. Seeds, Nat. Photonics 5, 416-419 (2011).

[2] S. Chen, W. Li, J. Wu, Q. Jiang, M. Tang, S. Shutts, S. Elliott, A. Sobiesierski, A. Seeds, I. Ross, P. Smowton and H. Liu, Nat. Photonics 10, 307-311 (2016).

[3] M. Liao, S. Chen, S. Hou, S. Chen, J. Wu, M. Tang, K. Kennedy, W. Li, S. Kumar, M. Martin, T. Baron, C. Jin, I. Ross, A. Seeds and H. Liu, IEEE JSTQE 23 (2017).

[4] S. Chen, M. Liao, M. Tang, J. Wu, M. Martin, T. Baron, A. Seeds and H. Liu, Opt. Express 25, 4632-4639 (2017).

[5] M. Tang, S. Chen, J. Wu, Q. Jiang, V.G. Dorogan, M. Benamara, Y.I. Mazur, G. J. Salamo, A. Seeds, and H. Liu, Opt. Express 22, $11528-$ 11535 (2014).

[6] T. Wang, H. Liu, A. Lee, F. Pozzi, and A. Seeds, Opt. Express 19(12), 11381-11386 (2011).

[7] A. Lee, Q. Jiang, M. Tang, A. Seeds, and H. Liu, Opt. Express 20, 22181-22187 (2012).

[8] S. Chen, M. Tang, J. Wu, Q. Jiang, V.G. Dorogan, M. Benamara, Y.I. Mazur, G.J. Salamo, P. Smowton, A. Seeds, and H. Liu, ACS

Photonics 1, 638-642 (2014). 